\title{
Slow motions of a circular cylinder experiencing slip near a plane wall
}

\author{
H.J. Keh*, L.R. Wang \\ Department of Chemical Engineering, National Taiwan University, Taipei 10617, Taiwan, ROC
}

Received 27 March 2007; accepted 6 November 2007

Available online 2 January 2008

\begin{abstract}
A theoretical study is presented for the two-dimensional creeping flow caused by a long circular cylindrical particle translating and rotating in a viscous fluid near a large plane wall parallel to its axis. The fluid is allowed to slip at the surface of the particle. The Stokes equations for the fluid velocity field are solved in the quasi-steady limit using cylindrical bipolar coordinates. Semi-analytical solutions for the drag force and torque acting on the particle by the fluid are obtained for various values of the slip coefficient associated with the particle surface and of the relative separation distance between the particle and the wall. The results indicate that the translation and rotation of the confined cylinder are not coupled with each other. For the motion of a no-slip cylinder near a plane wall, our hydrodynamic drag force and torque results reduce to the closed-form solutions available in the literature. The boundary-corrected drag force and torque acting on the particle decrease with an increase in the slip coefficient for an otherwise specified condition. The plane wall exerts the greatest drag on the particle when its migration occurs normal to it, and the least in the case of motion parallel to it. The enhancement in the hydrodynamic drag force and torque on a translating and rotating particle caused by a nearby plane wall is much more significant for a cylinder than for a sphere. (C) 2007 Elsevier Ltd. All rights reserved.
\end{abstract}

Keywords: Creeping flow; Circular cylinder; Slip-flow surface; Drag force and torque; Boundary effect

\section{Introduction}

The area of the movement of solid particles or fluid drops in a continuous medium at low Reynolds numbers is of much fundamental and practical interest in the fields of chemical, biochemical, and environmental engineering and science. The theoretical treatment of this subject has grown out of the classic work of Stokes (1851) for a translating rigid sphere in an unbounded viscous fluid. Oberbeck (1876) extended this result to the translation of an ellipsoid. More recently, analytical solutions of the creeping-flow problem have been obtained for rigid particles whose shapes correspond to a coordinate surface of one of the special orthogonal coordinate systems in which the Stokes equations are simply separable (Payne and Pell, 1960) and for long slender bodies (Batchelor, 1970). Explicit expressions for the resistance force and torque experienced by a rigid, slightly deformed sphere undergoing low-Reynolds-number translational and rotational motions in an unbounded fluid were also derived to the first order in the small parameter characterizing the deformation (Brenner, 1964).

\footnotetext{
*Corresponding author. Tel.: + 886233663048 ; fax: + 886223623040.

E-mail address: huan@ntu.edu.tw (H.J. Keh).
} 
Hadamard (1911) and Rybczynski (1911) extended independently the result of Stokes to the translation of a fluid sphere. Assuming continuous velocity and continuous tangential stress across the interface of fluid phases, they found that the force exerted on a spherical drop of radius $a$ by the surrounding fluid of viscosity $\eta$ is

$$
\mathbf{F}_{0}=-6 \pi \eta a \frac{3 \eta^{*}+2}{3 \eta^{*}+3} \mathbf{U}
$$

Here $\mathbf{U}$ is the migration velocity of the drop and $\eta^{*}$ is the internal-to-external viscosity ratio. Since the fluid properties are arbitrary, Eq. (1) degenerates to the case of translation of a solid sphere (Stokes' law) when $\eta^{*} \rightarrow \infty$ and to the case of motion of a gas bubble with spherical shape in the limit $\eta^{*} \rightarrow 0$.

In most practical applications, particles or drops are not isolated. So, it is important to determine if the presence of neighboring particles and/or boundaries significantly affects the movement of particles. Problems of the hydrodynamic interactions between two or more particles and between particles and boundaries for arbitrary values of $\eta^{*}$ have been treated extensively in the past. Summaries for the current state of knowledge in this area and some informative references can be found in Keh and Tseng (1992) and Chang and Keh (2006).

In the general formulation of the Stokes problem, it is usually assumed that no slippage arises at the solid-fluid interfaces. Actually, this is an idealization of the transport processes involved. The phenomena that the adjacent fluid can slip frictionally over a solid surface occur for cases such as the low-density gas flow surrounding an aerosol particle (Kennard, 1938; Hutchins et al., 1995), the aqueous liquid flow near a hydrophobic surface (Tretheway and Meinhart, 2002; Gogte et al., 2005), and the Newtonian fluid flow over a porous surface (Beavers and Joseph, 1967; Saffman, 1971; Nir, 1976), which have been confirmed, both experimentally and theoretically. Presumably, any such slipping would be proportional to the local tangential stress next to the solid surface (Happel and Brenner, 1983), known as the Navier slip (see Eq. (11b)), at least as long as the velocity gradient is small. The constant of proportionality, $\beta^{-1}$, is called a "slip coefficient."

Basset (1961) derived the following expressions for the force and torque exerted by the fluid on a translating and rotating rigid sphere with a slip-flow boundary condition at its surface

$$
\begin{aligned}
& \mathbf{F}_{0}=-6 \pi \eta a \frac{\beta a+2 \eta}{\beta a+3 \eta} \mathbf{U}, \\
& \mathbf{T}_{0}=-8 \pi \eta a^{3} \frac{\beta a}{\beta a+3 \eta} \mathbf{\Omega} .
\end{aligned}
$$

Here $\mathbf{U}$ and $\boldsymbol{\Omega}$ are the translational and angular velocities, respectively, of the particle. In the particular case of $\beta \rightarrow \infty$, there is no slip at the particle surface and Eq. (2a) degenerates to Stokes law. When $\beta=0$, there is a perfect slip at the particle surface and Eq. (2a) is identical to Eq. (1) taking $\eta^{*} \rightarrow 0$. Recently, the slip-surface creeping motions caused by a general axisymmetric particle translating along its axis of revolution (Keh and Huang, 2004) and by a slightly deformed sphere translating and rotating in arbitrary directions (Senchenko and Keh, 2006) have been theoretically examined.

The slip coefficient in Eq. (2) has been determined experimentally for various gas-solid systems and found to agree with the general kinetic theory of gases. It can be calculated from

$$
\frac{\eta}{\beta}=C_{\mathrm{m}} l,
$$

where $l$ is the mean free path of a gas molecule, and $C_{\mathrm{m}}$ is a dimensionless constant related to the momentum accommodation coefficient at the solid surface (Kennard, 1938). Although $C_{\mathrm{m}}$ surely depends upon the nature of the surface and adjacent fluid, an examination of the experimental data and theoretical predictions suggests that it will be in the range 1.0-1.5 (Davis, 1972; Talbot et al., 1980; Sharipov and Kalempa, 2003). The quantity $\eta / \beta$ is a length, which can be envisioned by noting that the fluid motion is the same as if the solid surface is displaced inward by a distance $\eta / \beta$ with the velocity gradient extending uniformly right up to no-slip velocity at the surface. The reciprocal of the factor $(\beta a+2 \eta) /(\beta a+3 \eta)$ in Eq. (2a) is equivalent to the so-called Cunningham correction factor for the slip effect.

The boundary effects on the motion of solid particles with finite values of $\beta a / \eta$ are different, both physically and mathematically, from those of fluid droplets of finite viscosities. Through an exact representation in spherical bipolar coordinates, Reed and Morrison (1974) and Chen and Keh (1995) examined the creeping motion of a rigid sphere normal to an infinite plane wall, where the fluid may slip at the solid surfaces. Later, the quasi-steady translation of a slip spherical particle in a spherical cavity was also theoretically studied (Keh and Chang, 1998; Lu and Lee, 2001). An analytical expression for the wall-corrected drag force exerted by the fluid on the particle located at the center of the cavity was derived in closed form. Recently, the slow translational and rotational motions of a slip sphere parallel 
(Chen and Keh, 2003) or perpendicular (Chang and Keh, 2006) to two parallel plane walls at an arbitrary position between them have been examined using the boundary collocation method. Numerical results for the hydrodynamic drag force and torque exerted on the particle were obtained as functions of $\beta a / \eta$ and the respective relative distances from the particle center to the two plane walls. However, the boundary effects on the creeping motion of a nonspherical slip particle have not been investigated yet.

The objective of this paper is to obtain solutions for the slow motion of a long circular cylindrical particle with a slip surface in an arbitrary transverse direction (the rotation of the particle about its axis is allowed) near a large plane wall parallel to its axis and to compare the results with those available for the corresponding motion of a sphere. The creeping flow equations applicable to the system are solved by using cylindrical bipolar coordinates and the wallcorrected drag force and torque acting on the particle are obtained for various cases. For the special case of movement of a particle with a zero slip coefficient, our results reduce to the available explicit expressions in the literature for the corresponding motion of a no-slip cylinder.

\section{Analysis}

We consider the two-dimensional creeping motion of a long circular cylindrical particle of radius $a$ in an incompressible Newtonian fluid near a large plane wall located at a distance $d$ from the axis of the cylinder at the quasisteady state. The fluid may slip at the surface of the particle but does not slip at the plane wall. The cylinder has a translational velocity $U_{x} \mathbf{e}_{x}+U_{y} \mathbf{e}_{y}$ and is rotating with an angular velocity $\Omega \mathbf{e}_{z}$, where $\mathbf{e}_{x}, \mathbf{e}_{y}$, and $\mathbf{e}_{z}$ are the unit vectors in rectangular coordinates $(x, y, z)$. The plane wall is stationary and the fluid is at rest far away from the particle.

In addition to rectangular coordinates, it is convenient to introduce cylindrical bipolar coordinates $(\xi, \psi, z)$, as illustrated in Fig. 1. The relationship between these two coordinate systems in any plane $z=$ constant is

$$
\begin{aligned}
& x=\frac{c \sinh \psi}{\cosh \psi-\cos \xi}, \\
& y=\frac{c \sin \xi}{\cosh \psi-\cos \xi},
\end{aligned}
$$

where $-\infty<\psi<\infty, 0 \leqslant \xi \leqslant 2 \pi$, and $c$ is a characteristic length in the bipolar coordinate system which is positive.

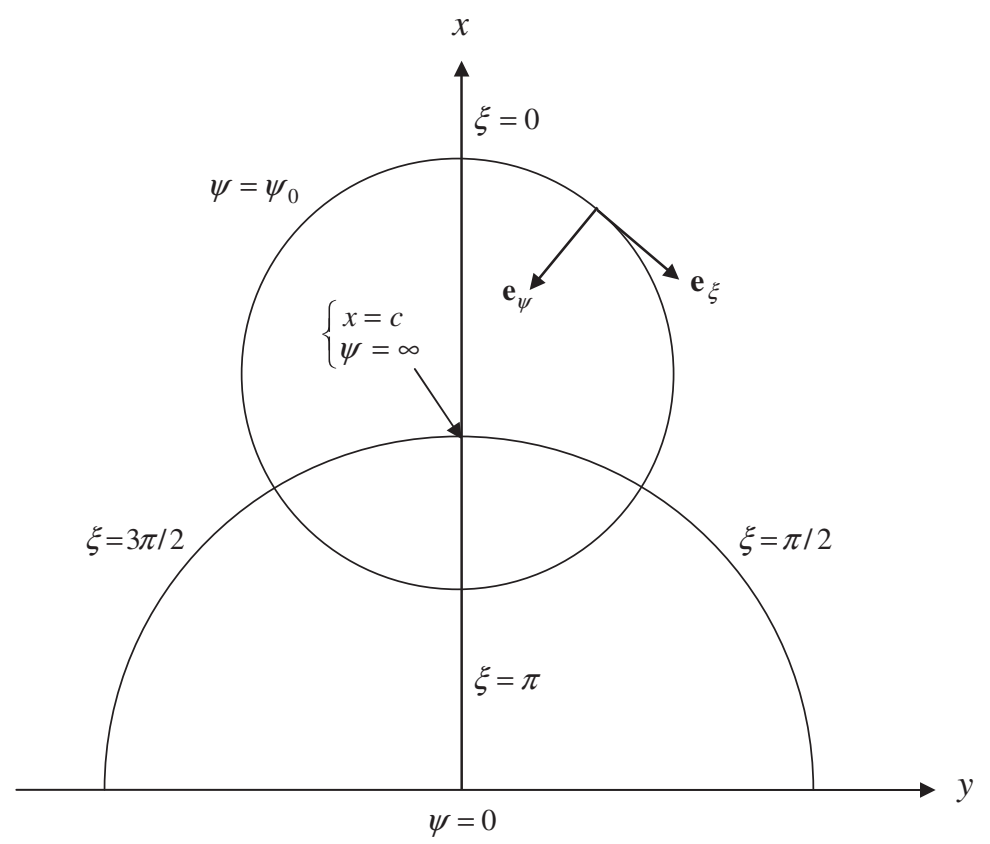

Fig. 1. Geometric sketch for the two-dimensional motion of a long circular cylinder near a large plane wall. 
The curves $\psi=$ constant correspond to a family of nonintersecting, coaxial circles (or cylinders) whose centers all lie along the $x$-axis. The special case $\psi=0$ generates a circle of infinite radius and corresponds to the entire $y$-axis (or the plane $x=0$ ). $\psi=\psi_{0}>0$ represents the circle (or the cylinder) of radius $a=c \operatorname{csch} \psi_{0}$, with its center at the point $\left(x=d=c \operatorname{coth} \psi_{0}, y=0\right)$. The ratio of the radius of the cylinder to the distance of the axis of the cylinder from the plane is related to $\psi_{0}$ by

$$
\lambda=a / d=\operatorname{sech} \psi_{0} .
$$

The creeping motion and continuity equations governing the fluid flow are

$$
\begin{aligned}
& \eta \nabla^{2} \mathbf{v}-\nabla p=\mathbf{0}, \\
& \nabla \cdot \mathbf{v}=0,
\end{aligned}
$$

where $\mathbf{v}$ is the fluid velocity distribution and $p$ is the dynamic pressure. Taking the curl of both sides of Eq. (6a) and introducing Eq. (6b) and the stream function $\Psi$ result in a fourth-order linear partial differential equation,

$$
\nabla^{4} \Psi=\nabla^{2}\left(\nabla^{2} \Psi\right)=0
$$

In Eqs. (6) and (7), the operators are

$$
\begin{aligned}
& \nabla=\mathbf{e}_{x} \frac{\partial}{\partial x}+\mathbf{e}_{y} \frac{\partial}{\partial y}=\frac{1}{c}(\cosh \psi-\cos \xi)\left(\mathbf{e}_{\xi} \frac{\partial}{\partial \xi}+\mathbf{e}_{\psi} \frac{\partial}{\partial \psi}\right), \\
& \nabla^{2}=\frac{\partial^{2}}{\partial x^{2}}+\frac{\partial^{2}}{\partial y^{2}}=\frac{1}{c^{2}}(\cosh \psi-\cos \xi)^{2}\left(\frac{\partial^{2}}{\partial \xi^{2}}+\frac{\partial^{2}}{\partial \psi^{2}}\right),
\end{aligned}
$$

where $\mathbf{e}_{\xi}$ and $\mathbf{e}_{\psi}$ are unit vectors in bipolar coordinates. Note that

$$
\begin{aligned}
& \mathbf{e}_{x}=\frac{1}{\cosh \psi-\cos \xi}\left[-\sinh \psi \sin \xi \mathbf{e}_{\xi}-(\cosh \psi \cos \xi-1) \mathbf{e}_{\psi}\right], \\
& \mathbf{e}_{y}=\frac{1}{\cosh \psi-\cos \xi}\left[(\cosh \psi \cos \xi-1) \mathbf{e}_{\xi}-\sinh \psi \sin \xi \mathbf{e}_{\psi}\right] .
\end{aligned}
$$

The stream function is related to the velocity components in bipolar coordinates by

$$
\begin{aligned}
& v_{\xi}=\frac{1}{c}(\cosh \psi-\cos \xi) \frac{\partial \Psi}{\partial \psi}, \\
& v_{\psi}=-\frac{1}{c}(\cosh \psi-\cos \xi) \frac{\partial \Psi}{\partial \xi} .
\end{aligned}
$$

The boundary conditions appropriate to the present problem require that

$$
\begin{aligned}
& \psi=0: \quad \mathbf{v}=\mathbf{0}, \\
& \psi=\psi_{0}: \quad \mathbf{v}=U_{x} \mathbf{e}_{x}+U_{y} \mathbf{e}_{y}+a \Omega \mathbf{e}_{\xi}-\frac{1}{\beta} \tau_{\psi \xi} \mathbf{e}_{\xi} .
\end{aligned}
$$

In Eq. (11b), $\tau_{\psi \xi}$ is the viscous stress in bipolar coordinates for the fluid and $1 / \beta$ is the frictional slip coefficient about the surface of the particle.

A general solution of the biharmonic Eq. (7) in bipolar coordinates, suitable for satisfying boundary conditions on the cylindrical particle and plane wall, has been given by Jeffery (1922) and Wakiya (1975),

$$
\begin{aligned}
\Psi= & c(\cosh \psi-\cos \xi)^{-1}[A \psi(\cosh \psi-\cos \xi)+(B+C \psi) \sinh \psi-D \psi \sin \xi \\
& +\sum_{n=1}^{\infty}\left\{\left[a_{n} \cosh (n+1) \psi+b_{n} \sinh (n+1) \psi+c_{n} \cosh (n-1) \psi+d_{n} \sinh (n-1) \psi\right] \cos n \xi\right. \\
& \left.\left.+\left[a_{n}^{\prime} \cosh (n+1) \psi+b_{n}^{\prime} \sinh (n+1) \psi+c_{n}^{\prime} \cosh (n-1) \psi+d_{n}^{\prime} \sinh (n-1) \psi\right] \sin n \xi\right\}\right] .
\end{aligned}
$$

Applying the boundary conditions given in Eqs. (11) and (12) and using Eqs. (9) and (10), one can obtain the following relations for the unknown coefficients $A, B, C, D, a_{n}, b_{n}, c_{n}, d_{n}, a_{n}^{\prime}, b_{n}^{\prime}, c_{n}^{\prime}$, and $d_{n}^{\prime}$ (in which $d_{1}$ and 
$d_{1}^{\prime}$ are trivial):

$$
\begin{aligned}
& A=\frac{a \Omega \cosh \psi_{0}}{\alpha}-\frac{U_{y}}{\sinh ^{2} \psi_{0}}-\frac{C\left(\alpha+C_{\mathrm{m}}^{*}+2 \alpha \psi_{0} / \cosh 2 \psi_{0}\right)}{\alpha \tanh \psi_{0}}, \\
& a_{1}=-\frac{A}{2} \tanh \psi_{0}-\frac{C \psi_{0}+U_{y}}{\sinh 2 \psi_{0}}, \\
& a_{2} K_{2}=4 A \alpha \sinh \psi_{0}+4\left(2 a_{1} \alpha-C C_{\mathrm{m}}^{*}\right) \cosh \psi_{0}, \\
& a_{n+1} K_{n+1}-2 a_{n}\left(H_{n}+K_{n} \cosh \psi_{0}\right)+a_{n-1} K_{n-1}=0 \quad(n \geqslant 2), \\
& B=-A, \quad b_{1}=\frac{A}{2}, \quad c_{n}=-a_{n} \quad(n \geqslant 1), \\
& b_{n}=a_{n} M_{n}, \quad d_{n}=\frac{1+n}{1-n} b_{n} \quad(n \geqslant 2), \\
& a_{1}^{\prime}=\frac{\left(2 \psi_{0}-\sinh _{2} \psi_{0}\right) D+2 U_{x}}{4 \sinh ^{2} \psi_{0}}, \\
& a_{2}^{\prime} K_{2}=4 D\left(\alpha+C_{\mathrm{m}}^{*}\right) \sinh \psi_{0}+8 a_{1}^{\prime} \alpha \cosh \psi_{0}, \\
& a_{n+1}^{\prime} K_{n+1}-2 a_{n}^{\prime}\left(H_{n}+K_{n} \cosh \psi_{0}\right)+a_{n-1}^{\prime} K_{n-1}=0 \quad(n \geqslant 2), \\
& b_{1}^{\prime}=\frac{1}{2} D, \quad c_{n}^{\prime}=-a_{n}^{\prime} \quad(n \geqslant 1), \\
& b_{n}^{\prime}=a_{n}^{\prime} M_{n}, \quad d_{n}^{\prime}=\frac{1+n}{1-n} b_{n}^{\prime} \quad(n \geqslant 2), \\
& \quad(n)
\end{aligned}
$$

where

$$
\begin{aligned}
& \alpha=\sinh ^{2} \psi_{0}+C_{\mathrm{m}}^{*} \cosh 2 \psi_{0}, \\
& K_{n}=\frac{4 n\left(\sinh 2 n \psi_{0}-n \sinh 2 \psi_{0}\right) C_{\mathrm{m}}^{*}}{(1-n) \sinh (n+1) \psi_{0}+(1+n) \sinh (n-1) \psi_{0}} \quad(n \geqslant 1), \\
& H_{n}=\frac{4\left(\sinh ^{2} n \psi_{0}-n^{2} \sinh ^{2} \psi_{0}\right) \sinh \psi_{0}}{(1-n) \sinh (n+1) \psi_{0}+(1+n) \sinh (n-1) \psi_{0}} \quad(n \geqslant 2), \\
& M_{n}=\frac{(1-n)\left[\cosh (n-1) \psi_{0}-\cosh (n+1) \psi_{0}\right]}{(1-n) \sinh (n+1) \psi_{0}+(1+n) \sinh (n-1) \psi_{0}} \quad(n \geqslant 2),
\end{aligned}
$$

and $C_{\mathrm{m}}^{*}=\eta / \beta a\left[=C_{\mathrm{m}} l / a\right.$ from Eq. (3) for an aerosol cylinder]. Because the coefficients $a_{n}$ (and also $b_{n}, c_{n}, d_{n}, a_{n}^{\prime}, b_{n}^{\prime}, c_{n}^{\prime}$, and $d_{n}^{\prime}$ ) should approach zero as $n \rightarrow \infty$ for the stream function to remain bounded, the coefficients $A$, $C$, and $a_{n}$ can be determined by simultaneously solving Eqs. (13a)-(13c) and the first $N$ equations of the recurrence relation (13d) provided that $N$ is sufficiently large that $a_{N+2}$ is negligible. Then, with the knowledge of $A$ and $a_{n}$, the coefficients $B, b_{n}$, $c_{n}$, and $d_{n}$ can be calculated using Eq. (14). Similarly, the coefficients $D$ and $a_{n}^{\prime}$ are to be determined by simultaneously solving Eqs. (15a) and (15b) and the first $N$ equations of the recurrence relation (15c), and then the coefficients $b_{n}^{\prime}, c_{n}^{\prime}$, and $d_{n}^{\prime}$ can be calculated using Eq. (16).

The drag force and torque per unit length exerted by the fluid on the cylinder can be determined from

$$
\begin{aligned}
& \mathbf{F}=-\int_{0}^{2 \pi}\left(\mathbf{e}_{\psi} \cdot \boldsymbol{\Pi}\right)_{\psi=\psi_{0}} \frac{a \sinh \psi_{0} \mathrm{~d} \xi}{\cosh \psi_{0}-\cos \xi}, \\
& \mathbf{T}=\int_{0}^{2 \pi}\left[\mathbf{e}_{\psi} \times\left(\mathbf{e}_{\psi} \cdot \boldsymbol{\Pi}\right)\right]_{\psi=\psi_{0}} \frac{a^{2} \sinh \psi_{0} \mathrm{~d} \xi}{\cosh \psi_{0}-\cos \xi},
\end{aligned}
$$


respectively. In Eq. (19), $\Pi$ is the total stress tensor,

$$
\boldsymbol{\Pi}=-p \mathbf{I}+\eta\left[\nabla \mathbf{v}+(\nabla \mathbf{v})^{\mathrm{T}}\right],
$$

where I is the unit dyadic. Substituting Eq. (12) into Eq. (19) and using Eqs. (8a), (10), and (20) yield

$$
\begin{aligned}
& \mathbf{F}=F_{x} \mathbf{e}_{x}+F_{y} \mathbf{e}_{y}=4 \pi \eta\left(D \mathbf{e}_{x}+C \mathbf{e}_{y}\right), \\
& \mathbf{T}=T \mathbf{e}_{z}=-4 \pi \eta a\left(A \sinh \psi_{0}+C \cosh \psi_{0}\right) \mathbf{e}_{z},
\end{aligned}
$$

where the coefficients $A, C$, and $D$ have been obtained using Eqs. (13) and (15).

In the limiting case of $C_{\mathrm{m}}^{*}=0$ or $\beta a / \eta \rightarrow \infty$, there is no slip at the particle surface and Eqs. (13) and (15) result in

$$
A=\left(\frac{U_{y}}{\psi_{0}}+\frac{a \Omega}{\sinh \psi_{0}}\right) \operatorname{coth} \psi_{0}, \quad C=-\frac{U_{y}}{\psi_{0}}, \quad D=-\frac{U_{x}}{\psi_{0}-\tanh \psi_{0}} .
$$

Substituting Eq. (22) into Eq. (21), one obtains

$$
\begin{aligned}
& \mathbf{F}=-4 \pi \eta\left(\frac{U_{x}}{\psi_{0}-\tanh \psi_{0}} \mathbf{e}_{x}+\frac{U_{y}}{\psi_{0}} \mathbf{e}_{y}\right), \\
& \mathbf{T}=-4 \pi \eta a^{2} \Omega \operatorname{coth} \psi_{0} \mathbf{e}_{z},
\end{aligned}
$$

where the expressions for the hydrodynamic force and torque previously obtained (Keh et al., 1991) is reproduced.

In the other limiting case of $C_{\mathrm{m}}^{*} \rightarrow \infty$ or $\beta a / \eta=0$, there is a perfect slip at the particle surface and Eqs. (13) and (15) lead to

$$
A=\frac{2 U_{y} \operatorname{coth} \psi_{0}}{2 \psi_{0}+\tanh 2 \psi_{0}}, \quad C=-\frac{2 U_{y}}{2 \psi_{0}+\tanh 2 \psi_{0}}, \quad D=-\frac{2 U_{x}}{2 \psi_{0}-\tanh 2 \psi_{0}} .
$$

Substituting Eq. (24) into Eq. (21), one obtains

$$
\begin{aligned}
& \mathbf{F}=-4 \pi \eta\left(\frac{2 U_{x}}{2 \psi_{0}-\tanh 2 \psi_{0}} \mathbf{e}_{x}+\frac{2 U_{y}}{2 \psi_{0}+\tanh 2 \psi_{0}} \mathbf{e}_{y}\right), \\
& \mathbf{T}=\mathbf{0} .
\end{aligned}
$$

As expected, a particle with a perfect slip surface bears no hydrodynamic torque.

For the translational streaming motion of an infinitely long circular cylinder perpendicular to its axis in an unbounded fluid, i.e. the case $\lambda=0$ or $\psi_{0} \rightarrow \infty$, there exists no solution of Eq. (7) (known as the Stokes paradox) and both the force components $F_{x}$ and $F_{y}$ vanish, regardless of the slip parameter $C_{\mathrm{m}}^{*}$. On the other hand, for the rotational motion of an infinitely long circular cylinder about its axis in an unbounded fluid, Eq. (21b) reduces to closed form as

$$
\mathbf{T}_{0}=T_{0} \mathbf{e}_{z}=-4 \pi \eta a^{2} \Omega \frac{1}{1+2 C_{\mathrm{m}}^{*}} \mathbf{e}_{z} .
$$

Again, Eq. (26) leads to $T_{0}=0$ for a perfect slip particle (with $C_{\mathrm{m}}^{*} \rightarrow \infty$ ).

\section{Results and discussion}

We consider the two-dimensional quasi-steady creeping flow produced by an infinitely long, slip-surface cylinder (at $\psi=\psi_{0}$ ) of radius $a$ translating with velocity $U_{x} \mathbf{e}_{x}+U_{y} \mathbf{e}_{y}$ and rotating with angular velocity $\Omega \mathbf{e}_{z}$ near an infinite plane wall (at $x=0$ ). Through the numerical solution of Eqs. (13) and (15) using a personal computer, the coefficients $A, C$, and $D$ together with $a_{n}$ and $a_{n}^{\prime}$ of the stream function expressed by Eq. (12) have been determined for various values of the parameters $C_{\mathrm{m}}^{*}$ and $\lambda$. For all the cases, $N$ equal to about 250 was sufficiently large that the $(N+1)$ th terms of the series of coefficients $a_{n}$ and $a_{n}^{\prime}$ are negligible and increases in $N$ do not alter the calculated values of all the coefficients appreciably. With the knowledge of the coefficients $A, C$, and $D$, the hydrodynamic force and torque acting on the cylinder per unit length can be calculated using Eq. (21). The results indicate that the translation and rotation of the cylinder for this creeping motion are not coupled with each other; namely, the force and torque components $F_{x}, F_{y}$, and $T$ depend only on $U_{x}, U_{y}$, and $\Omega$, respectively. This behavior is different from that for the creeping translational and rotational motions of a slip-surface sphere in the vicinity of a plane wall (Chen and Keh, 2003). 
Table 1

The dimensionless drag force $-F_{x} / 4 \pi \eta U_{x}$ experienced by a slip circular cylinder translating transversally and normal to a plane wall at various values of $\lambda$ and $C_{\mathrm{m}}^{*}$

\begin{tabular}{|c|c|c|c|c|c|}
\hline \multirow[t]{2}{*}{$\lambda$} & \multicolumn{5}{|l|}{$-F_{x} / 4 \pi \eta U_{x}$} \\
\hline & $C_{\mathrm{m}}^{*}=0$ & $C_{\mathrm{m}}^{*}=0.01$ & $C_{\mathrm{m}}^{*}=0.1$ & $C_{\mathrm{m}}^{*}=1$ & $C_{\mathrm{m}}^{*} \rightarrow \infty$ \\
\hline 0.001 & 0.15149 & 0.15127 & 0.14961 & 0.14421 & 0.14083 \\
\hline 0.01 & 0.23265 & 0.23212 & 0.22822 & 0.21591 & 0.20841 \\
\hline 0.1 & 0.50044 & 0.49801 & 0.48053 & 0.42945 & 0.40109 \\
\hline 0.2 & 0.76183 & 0.75630 & 0.71746 & 0.61203 & 0.55787 \\
\hline 0.3 & 1.08710 & 1.07618 & 1.00173 & 0.81577 & 0.72760 \\
\hline 0.4 & 1.53779 & 1.51691 & 1.37995 & 1.07020 & 0.93572 \\
\hline 0.5 & 2.21763 & 2.17690 & 1.92346 & 1.41592 & 1.21642 \\
\hline 0.6 & 3.34882 & 3.26400 & 2.77394 & 1.93219 & 1.63718 \\
\hline 0.7 & 5.51130 & 5.31008 & 4.27054 & 2.81195 & 2.36605 \\
\hline 0.8 & 10.73570 & 10.11742 & 7.47247 & 4.68661 & 3.96872 \\
\hline 0.9 & 31.99446 & 28.39274 & 18.06223 & 11.29337 & 9.91559 \\
\hline 0.95 & 92.70814 & 74.49890 & 42.11408 & 28.31604 & 25.95631 \\
\hline 0.975 & 265.29155 & 184.18642 & 99.19621 & 74.08797 & 70.30084 \\
\hline 0.99 & 1055.87595 & 569.28369 & 326.42637 & 276.97007 & 270.30334 \\
\hline 0.995 & 2993.24207 & 1314.39280 & 845.25628 & 767.22778 & 757.28960 \\
\hline 0.999 & 33525.92266 & 10231.19869 & 8627.03667 & 8425.40680 & 8401.59593 \\
\hline
\end{tabular}

Table 2

The dimensionless drag force $-F_{y} / 4 \pi \eta U_{y}$ experienced by a slip circular cylinder translating transversally and parallel to a plane wall at various values of $\lambda$ and $C_{\mathrm{m}}^{*}$

\begin{tabular}{|c|c|c|c|c|c|}
\hline \multirow[t]{2}{*}{$\lambda$} & \multicolumn{5}{|c|}{$-F_{y} 4 \pi \eta U_{y}$} \\
\hline & $C_{\mathrm{m}}^{*}=0$ & $C_{\mathrm{m}}^{*}=0.01$ & $C_{\mathrm{m}}^{*}=0.1$ & $C_{\mathrm{m}}^{*}=1$ & $C_{\mathrm{m}}^{*} \rightarrow \infty$ \\
\hline 0.001 & 0.13156 & 0.13139 & 0.13014 & 0.12604 & 0.12344 \\
\hline 0.01 & 0.18874 & 0.18839 & 0.18582 & 0.17757 & 0.17247 \\
\hline 0.1 & 0.33409 & 0.33299 & 0.32501 & 0.30058 & 0.28627 \\
\hline 0.2 & 0.43622 & 0.43432 & 0.42070 & 0.38065 & 0.35812 \\
\hline 0.3 & 0.53367 & 0.53076 & 0.51021 & 0.45248 & 0.42136 \\
\hline 0.4 & 0.63824 & 0.63393 & 0.60410 & 0.52492 & 0.48428 \\
\hline 0.5 & 0.75933 & 0.75290 & 0.70977 & 0.60377 & 0.55193 \\
\hline 0.6 & 0.91024 & 0.90030 & 0.83661 & 0.69417 & 0.63035 \\
\hline 0.7 & 1.11658 & 1.10002 & 1.00107 & 0.80860 & 0.73071 \\
\hline 0.8 & 1.44270 & 1.41052 & 1.24063 & 0.97476 & 0.88158 \\
\hline 0.9 & 2.14066 & 2.04913 & 1.68521 & 1.30676 & 1.19985 \\
\hline 0.95 & 3.09563 & 2.85659 & 2.20376 & 1.75587 & 1.64596 \\
\hline 0.975 & 4.42528 & 3.85317 & 2.86249 & 2.39310 & 2.28454 \\
\hline 0.99 & 7.04154 & 5.48658 & 4.13000 & 3.67308 & 3.56723 \\
\hline 0.995 & 9.97914 & 7.04912 & 5.56780 & 5.12707 & 5.02266 \\
\hline 0.999 & 22.35136 & 13.11485 & 11.71123 & 11.29345 & 11.19057 \\
\hline
\end{tabular}

The numerical solutions for the dimensionless hydrodynamic force and torque components $-F_{x} / 4 \pi \eta U_{x},-F_{y} / 4 \pi \eta U_{y}$, and $T / T_{0}$ [where $T_{0}$ is defined by Eq. (26)] are presented in Tables $1-3$ for various values of the parameters $C_{\mathrm{m}}^{*}$ and $\lambda$ and plotted versus $C_{\mathrm{m}}^{*}$ in Figs. 2-4 for different values of $\lambda$. All of the results obtained converge satisfactorily to at least the significant digits shown in these tables. As expected, each of the force components is a monotonically increasing function of $\lambda$, and will become infinite in the limit $\lambda=1$ for any given value of $C_{\mathrm{m}}^{*}$. Although the normalized torque $T / T_{0}$ for the case of no $\operatorname{slip}\left(C_{\mathrm{m}}^{*}=0\right)$ increases with an increase in $\lambda$, it is not a monotonic function of $\lambda$ for the case of finite values of $C_{\mathrm{m}}^{*}\left(T / T_{0}\right.$ increases with an increase in $\lambda$ from unity at $\lambda=0$, reaches a maximum at a value of $\lambda$ greater 
Table 3

The normalized hydrodynamic torque $T / T_{0}$ experienced by a slip circular cylinder rotating about its axis parallel to a plane wall at various values of $\lambda$ and $C_{\mathrm{m}}^{*}$

\begin{tabular}{|c|c|c|c|c|c|}
\hline \multirow[t]{2}{*}{$\lambda$} & \multicolumn{5}{|l|}{$T / T_{0}$} \\
\hline & $C_{\mathrm{m}}^{*}=0$ & $C_{\mathrm{m}}^{*}=0.01$ & $C_{\mathrm{m}}^{*}=0.1$ & $C_{\mathrm{m}}^{*}=1$ & $C_{\mathrm{m}}^{*}=10$ \\
\hline 0.1 & 1.00504 & 1.00494 & 1.00419 & 1.00167 & 1.00024 \\
\hline 0.2 & 1.02062 & 1.02020 & 1.01710 & 1.00675 & 1.00096 \\
\hline 0.3 & 1.04828 & 1.04727 & 1.03977 & 1.01544 & 1.00217 \\
\hline 0.4 & 1.09109 & 1.08906 & 1.07426 & 1.02809 & 1.00390 \\
\hline 0.5 & 1.15470 & 1.15095 & 1.12416 & 1.04527 & 1.00618 \\
\hline 0.6 & 1.25000 & 1.24318 & 1.19593 & 1.06777 & 1.00903 \\
\hline 0.7 & 1.40028 & 1.38733 & 1.30191 & 1.09674 & 1.01251 \\
\hline 0.8 & 1.66667 & 1.63857 & 1.46900 & 1.13370 & 1.01659 \\
\hline 0.9 & 2.29416 & 2.20527 & 1.77044 & 1.17998 & 1.02119 \\
\hline 0.95 & 3.20256 & 2.95276 & 2.03906 & 1.20601 & 1.02350 \\
\hline 0.975 & 4.50035 & 3.85669 & 2.23262 & 1.21875 & 1.02456 \\
\hline 0.99 & 7.08881 & 5.12512 & 2.37129 & 1.22557 & 1.02510 \\
\hline 0.995 & 10.01252 & 5.94136 & 2.41764 & 1.22748 & 1.02526 \\
\hline 0.999 & 22.36627 & 6.90824 & 2.44965 & 1.22873 & 1.02533 \\
\hline 0.9999 & 70.71245 & 7.12835 & 2.45473 & 1.21832 & 0.98001 \\
\hline 0.99999 & 223.60736 & 7.14260 & 2.40974 & 0.86148 & 0.54613 \\
\hline
\end{tabular}

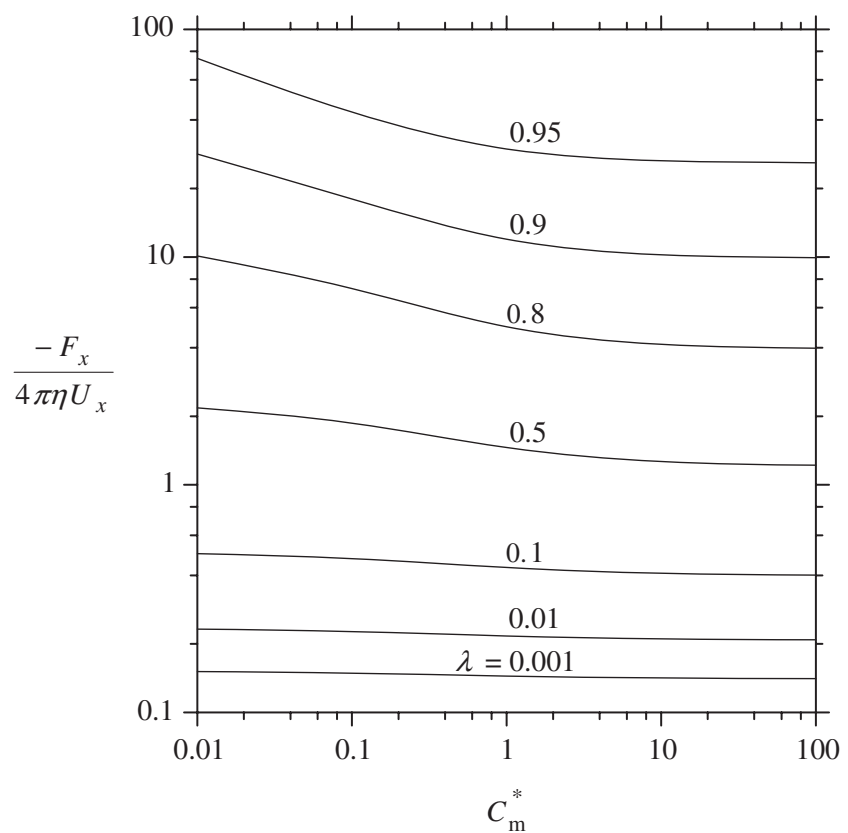

Fig. 2. Plots of the dimensionless drag force $-F_{x} / 4 \pi \eta U_{x}$ for the transverse translation of a circular cylinder normal to a plane wall versus the slip parameter $C_{\mathrm{m}}^{*}$ with $\lambda$ as a parameter.

than about 0.999 , and then decreases with a further increase in $\lambda$ ). In the limit $C_{\mathrm{m}}^{*} \rightarrow \infty$, the normalized torque $T / T_{0} \rightarrow 1$ (with $T \rightarrow 0$ and $T_{0} \rightarrow 0$ ) unless the value of $\lambda$ is very close to unity. For any given value of $\lambda$, these force and torque components decrease with an increase in the slip parameter $C_{\mathrm{m}}^{*}$ (or with a decrease in the coefficient $\beta$ ).

A comparison between the results of $-F_{x} / 4 \pi \eta U_{x}$ and $-F_{y} / 4 \pi \eta U_{y}$ indicates that, as expected, the plane wall exerts the most influence (or greatest drag) on the particle when its migration occurs normal to it, and the least in the case of motion parallel to it. The difference can be quite significant when the spacing between the particle and the confining 


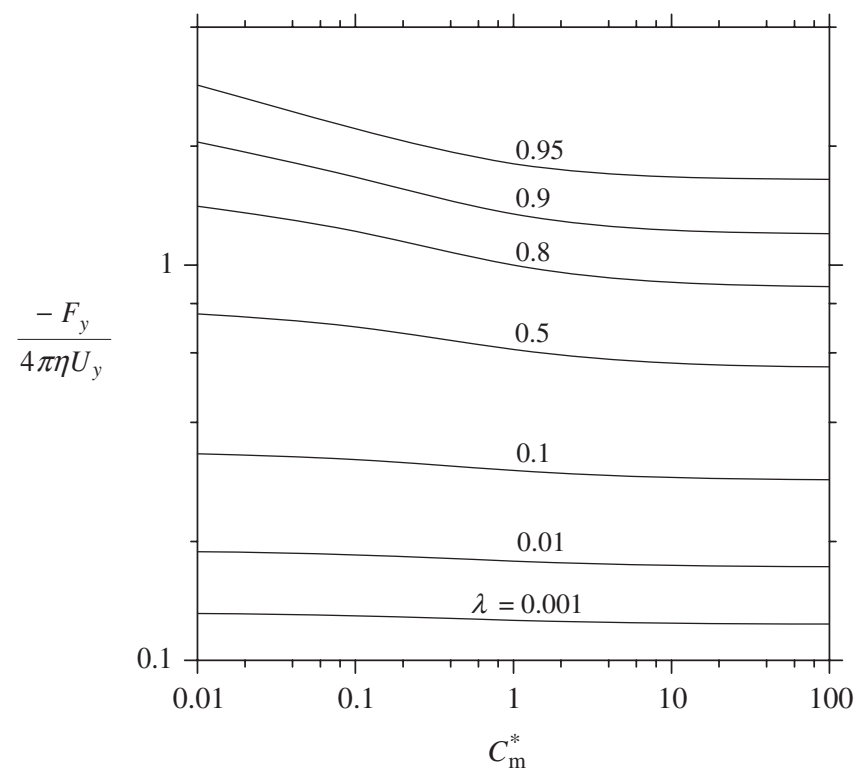

Fig. 3. Plots of the dimensionless drag force $-F_{y} / 4 \pi \eta U_{y}$ for the transverse translation of a circular cylinder parallel to a plane wall versus the slip parameter $C_{\mathrm{m}}^{*}$ with $\lambda$ as a parameter.

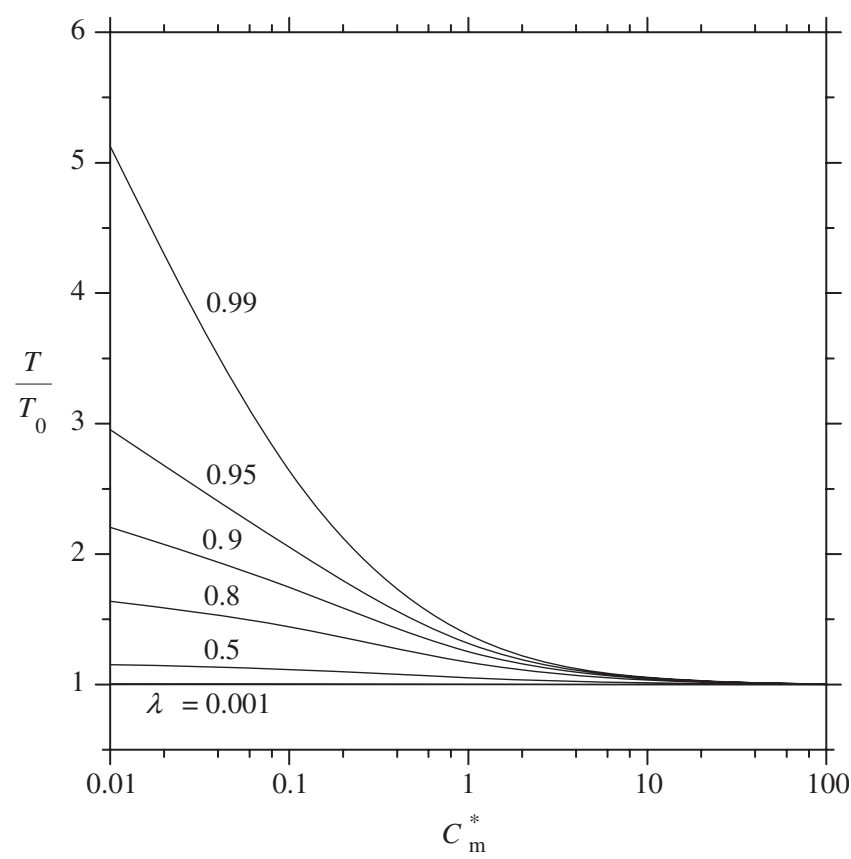

Fig. 4. Plots of the normalized torque $T / T_{0}$ for the rotation of a circular cylinder about its axis near a plane wall versus the slip parameter $C_{\mathrm{m}}^{*}$ with $\lambda$ as a parameter.

boundary is small. Therefore, the direction of transverse migration of a cylinder in the vicinity of a plane wall parallel to its axis is different from that of the applied force, except when the force is oriented parallel or perpendicular to the plane wall.

The quasi-steady motion of a slip sphere of radius $a$ with a translational velocity $U_{y}$ and angular velocity $\Omega$ parallel to an infinite plane wall at a distance $d$ from the particle center was investigated by Chen and Keh (2003) and its motion with a translational velocity $U_{x}$ normal to a plane wall was examined by Chang and Keh (2006) using a boundary 


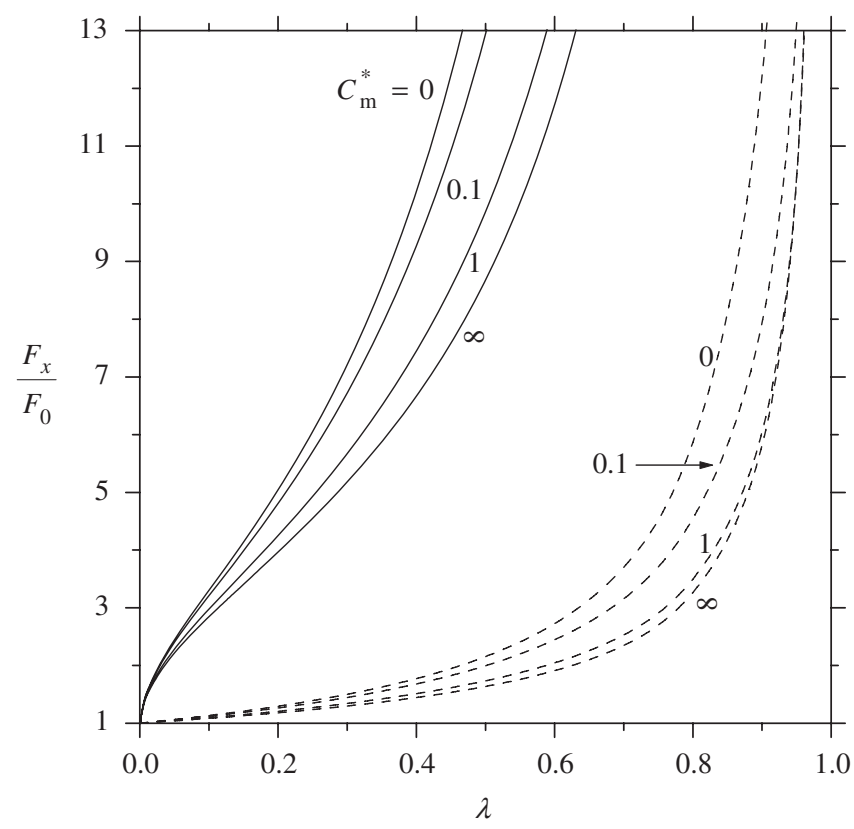

Fig. 5. Plots of the normalized drag force $F_{x} / F_{0}$ for the transverse translation of a circular cylinder normal to a plane wall versus the separation parameter $\lambda$ with $C_{\mathrm{m}}^{*}$ as a parameter. The dashed curves are plotted for the corresponding translation of a slip sphere for comparison.

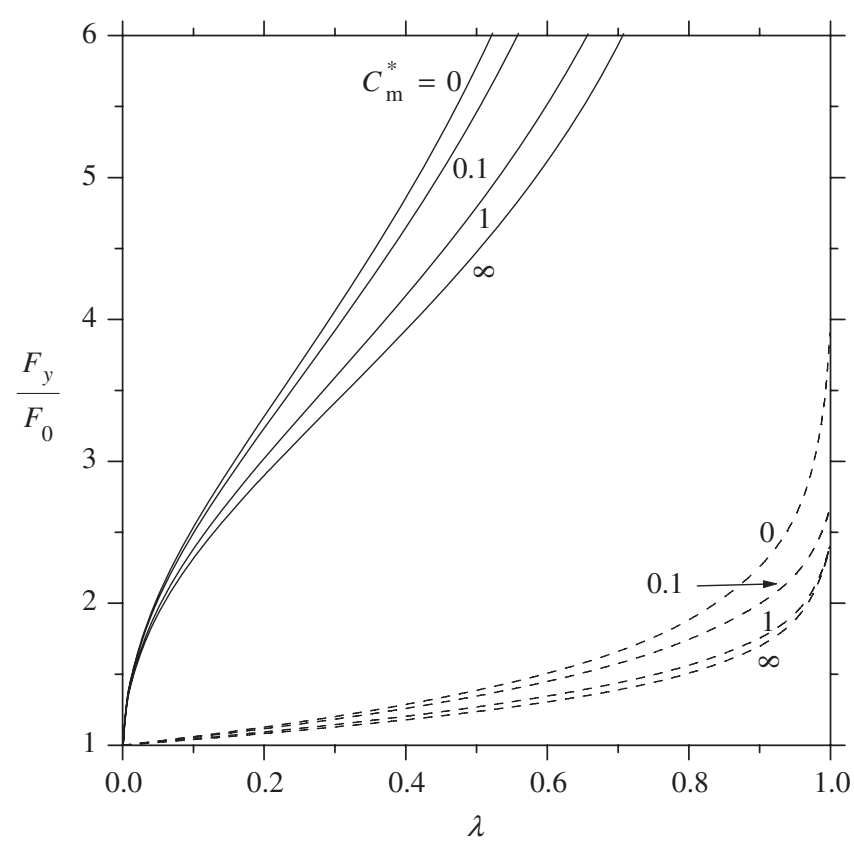

Fig. 6. Plots of the normalized drag force $F_{y} / F_{0}$ for the transverse translation of a circular cylinder parallel to a plane wall versus the separation parameter $\lambda$ with $C_{\mathrm{m}}^{*}$ as a parameter. The dashed curves are plotted for the corresponding translation of a slip sphere for comparison.

collocation method. In Figs. 5-7, their numerical solutions for the relevant normalized force and torque components $F_{x} / F_{0}, F_{y} / F_{0}$, and $T / T_{0}$ [where $F_{0}$ and $T_{0}$ are given by Eq. (2) for the motion of a slip sphere in an unbounded fluid], respectively, are plotted versus $\lambda(=a / d)$ for different values of $C_{\mathrm{m}}^{*}$ by dashed curves. For comparison, our solutions of 


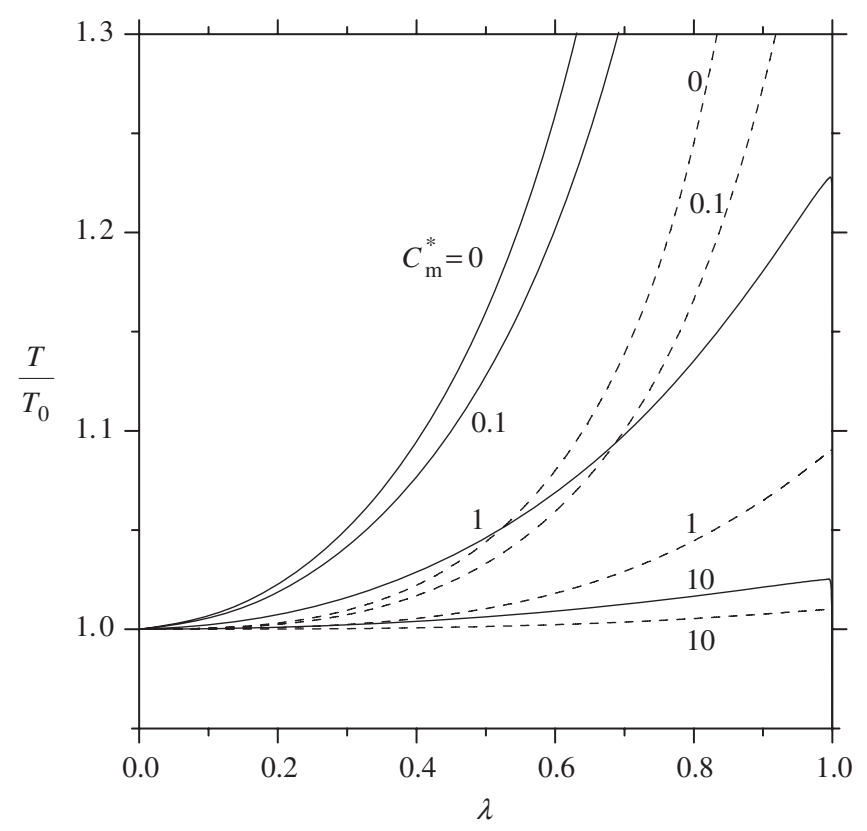

Fig. 7. Plots of the normalized torque $T / T_{0}$ for the rotation of a circular cylinder about its axis near a plane wall versus the separation parameter $\lambda$ with $C_{\mathrm{m}}^{*}$ as a parameter. The dashed curves are plotted for the corresponding rotation of a slip sphere for comparison.

the corresponding force and torque components for the case of two-dimensional motion of a slip circular cylinder near an infinite plane wall are also drawn by solid curves in the same figures. Since there exists no creeping-flow solution of $F_{0}$ for the transverse translation of a circular cylinder as $\lambda=0$, the hydrodynamic forces $F_{x}$ and $F_{y}$ acting on the slip cylinder with $\lambda=0.001$ are chosen to be the reference force $F_{0}$ for its corresponding motions in the proximity of the plane wall. As expected, Figs. 5-7 indicate that the enhancement in the hydrodynamic drag force and torque on a translating and rotating particle caused by a confining plane wall is much more significant for a cylinder than for a sphere for a given combination of the parameters $C_{\mathrm{m}}^{*}$ and $\lambda$.

\section{Concluding remarks}

In this work, the arbitrary two-dimensional translation and rotation of a long circular cylinder in the proximity of a large plane wall at the quasi-steady state are studied theoretically, where the ambient fluid may slip at the surface of the cylinder. A cylindrical bipolar coordinate system has been used to solve the Stokes equations for the fluid flow field. The results for the hydrodynamic drag force and torque exerted on the cylinder are obtained for various cases of the slip coefficient and of the separation between the cylinder and the plane wall. It has been found that, for a given relative position of the wall, the wall-corrected drag force and torque acting on the cylinder by the surrounding fluid are decreasing functions of the slip parameter $C_{\mathrm{m}}^{*}$.

In Tables 1-3 and Figs. 2-7, we present only the results for resistance problems, defined as those in which the drag force components $F_{x}$ and $F_{y}$ as well as torque $T$ exerted by the surrounding fluid on the particle translating and rotating near the confining wall are to be determined for specified particle velocities $U_{x}, U_{y}$, and $\Omega\left[=-T_{0}\left(1+2 C_{\mathrm{m}}^{*}\right) / 4 \pi \eta a^{2}\right.$ as given by Eq. (26)]. In a mobility problem, on the other hand, the external force components $F_{x}$ and $F_{y}$ as well as torque $T$ imposed on the particle are specified [with $T=4 \pi \eta a^{2} \Omega_{0} /\left(1+2 C_{\mathrm{m}}^{*}\right)$, where $\Omega_{0}$ will be the resulting angular velocity of the particle in the absence of the wall] and the boundary-corrected particle velocities $U_{x}, U_{y}$, and $\Omega$ are to be determined. For the twodimensional creeping motion of a long circular cylinder with an arbitrary slip coefficient near a large plane wall considered in this work, the translation and rotation are not coupled with each other, and thus, the ratios $4 \pi \eta U_{x} / F_{x}, 4 \pi \eta U_{y} / F_{y}$, and $\Omega / \Omega_{0}$ for a mobility problem are equal to the ratios $\left(-F_{x} / 4 \pi \eta U_{x}\right)^{-1},\left(-F_{y} / 4 \pi \eta U_{y}\right)^{-1}$, and $\left(T / T_{0}\right)^{-1}$, respectively, for its corresponding resistance problem. Thus, our results can also be applied to physical problems in which the applied force and torque on the cylinder are the prescribed quantities and the cylinder must move accordingly.

It is worth repeating that the value of the slip coefficient $C_{\mathrm{m}}$ for aerosol systems varies in a small range 1.0-1.5. For the continuum with slippage analysis to be valid for the rarefied gas flow surrounding a solid particle, the Knudsen 
number $(l / a)$ should be small (say, less than 1). Consequently, the parameter $C_{\mathrm{m}}^{*}$ can be any value less than the order unity for the case of motion of an aerosol particle. On the other hand, our result can also apply to the cases of the aqueous liquid flow near a hydrophobic surface and of the fluid flow over a porous surface, in which the range of the slip parameter $C_{\mathrm{m}}^{*}$ can be much wider and more experimental data for different situations are still needed. Therefore, in the previous section, we have chosen to present the numerical values of the hydrodynamic drag force and torque exerted on the particle for the parameter $C_{\mathrm{m}}^{*}$ with values from zero to infinity to provide a complete comparison, which is of fundamental interest and can be used for various cases in practice.

\section{Acknowledgment}

Part of this research was supported by the National Science Council of the Republic of China.

\section{References}

Basset, A.B., 1961. A Treatise on Hydrodynamics, vol. 2. Dover, New York.

Batchelor, G.K., 1970. Slender-body theory for particles of arbitrary cross-section in Stokes flow. Journal of Fluid Mechanics 44, 419-440.

Beavers, G.S., Joseph, D.D., 1967. Boundary conditions at a naturally permeable wall. Journal of Fluid Mechanics 30, $197-207$.

Brenner, H., 1964. The Stokes resistance of a slightly deformed sphere. Chemical Engineering Science 19, 519-539.

Chang, Y.C., Keh, H.J., 2006. Slow motion of a slip spherical particle perpendicular to two plane walls. Journal of Fluids and Structures 22, 647-661.

Chen, P.Y., Keh, H.J., 2003. Slow motion of a slip spherical particle parallel to one or two plane walls. Journal of the Chinese Institute of Chemical Engineers 34, 123-133.

Chen, S.H., Keh, H.J., 1995. Axisymmetric motion of two spherical particles with slip surfaces. Journal of Colloid and Interface Science 171, 63-72.

Davis, M.H., 1972. Collisions of small cloud droplets: gas kinetic effects. Journal of Atmospheric Science 29, 911-915.

Gogte, S., Vorobieff, P., Truesdell, R., Mammoli, A., van Swol, F., Shah, P., Brinker, C.J., 2005. Effective slip on textured superhydrophobic surfaces. Physics of Fluids 17, 051701-051714.

Hadamard, J.S., 1911. Mouvement permanent lent d'une sphere liquid et visqueuse dans un liquide visqueux, Comptes Rendus Hebdomadaires des Seances de V Academie des Sciences, Paris, 152, pp. 1735-1738.

Happel, J., Brenner, H., 1983. Low Reynolds Number Hydrodynamics. Nijhoff, Dordrecht, The Netherlands.

Hutchins, D.K., Harper, M.H., Felder, R.L., 1995. Slip correction measurements for solid spherical particles by modulated dynamic light scattering. Aerosol Science and Technology 22, 202-218.

Jeffery, G.B., 1922. The rotation of two circular cylinders in a viscous fluid. Proceedings of the Royal Society of London A 101, $169-174$.

Keh, H.J., Chang, J.H., 1998. Boundary effects on the creeping-flow and thermophoretic motions of an aerosol particle in a spherical cavity. Chemical Engineering Science 53, 2365-2377.

Keh, H.J., Huang, C.H., 2004. Slow motion of axisymmetric slip particles along their axes of revolution. International Journal of Engineering Science 42, 1621-1644.

Keh, H.J., Tseng, Y.K., 1992. Slow motion of multiple droplets in arbitrary three-dimensional configurations. AIChE Journal 38, 1881-1904.

Keh, H.J., Horng, K.D., Kuo, J., 1991. Boundary effects on electrophoresis of colloidal cylinders. Journal of Fluid Mechanics 231, 211-228.

Kennard, E.H., 1938. Kinetic Theory of Gases. McGraw-Hill, New York.

Lu, S.Y., Lee, C.T., 2001. Boundary effects on creeping motion of an aerosol particle in a non-concentric pore. Chemical Engineering Science 56, 5207-5216.

Nir, A., 1976. Linear shear flow past a porous particle. Applied Scientific Research 32, 313-325.

Oberbeck, A., 1876. Über stationäre Flussigkeitsbewegungen mit Berucksichtigung der inner Reibung. J. Reine Angewandte Mathematik 81, 62-80.

Payne, L.E., Pell, W.H., 1960. The Stokes flow problem for a class of axially symmetric bodies. Journal of Fluid Mechanics 7, 529-549.

Reed, L.D., Morrison, F.A., 1974. Particle interactions in viscous flow at small values of Knudsen number. Journal of Aerosol Science 5, 175-189.

Rybczynski, W., 1911. Über die fortschreitende bewegung einer flussigen kugel in einem zahenmedium. Bulletin of the Academic of Science Cracovie Series A 1, 40-46.

Saffman, P.G., 1971. On the boundary condition at the surface of a porous medium. Studies in Applied Mathematics 50, 93-101.

Senchenko, S., Keh, H.J., 2006. Slipping Stokes flow around a slightly deformed sphere. Physics of Fluids 18, 088104-1-4. 
Sharipov, F., Kalempa, D., 2003. Velocity slip and temperature jump coefficients for gaseous mixtures. I. Viscous slip coefficient. Physics of Fluids 15, 1800-1806.

Stokes, G.G., 1851. On the effect of the internal friction of fluid on pendulums. Transactions of the Cambridge Philosophical Society 9 , 8-106.

Talbot, L., Cheng, R.K., Schefer, R.W., Willis, D.R., 1980. Thermophoresis of particles in heated boundary layer. Journal of Fluid Mechanics 101, 737-758.

Tretheway, D.C., Meinhart, C.D., 2002. Apparent fluid slip at hydrophobic microchannel walls. Physics of Fluids 14, L9-L12.

Wakiya, S., 1975. Application of bipolar coordinates to the two-dimensional creeping motion of a liquid. II. Some problems for two circular cylinders in viscous fluid. Journal of the Physical Society of Japan 39, 1603-1607. 